\title{
Rotational periodicities in sunspot relative numbers ${ }^{\star}$
}

\author{
H. Balthasar
}

Astrophysikalisches Institut Potsdam, An der Sternwarte 16, 14482 Potsdam, Germany

e-mail: hbalthasar@aip.de

Received 14 March 2007 / Accepted 10 May 2007

\section{ABSTRACT}

\begin{abstract}
Context. The search for active longitudes on the Sun has a long history, and many controversial results have been published. Recently the question became more important when active longitudes were found on other stars.

Aims. The aim of this paper is to investigate an integral measure of solar activity available for a long time interval and which allows enough frequency resolution for the investigation of active longitudes. Such a measure is given by the daily sunspot relative numbers. Methods. A search for periodicities is performed with a classical Fast Fourier Transform (FFT), with a wavelet analysis and with the tool of superimposed epochs.

Results. The FFT yields a hump of power peaks near the synodic rotation period of 27 days, but only a very weak and insignificant enhancement around 13.5 days, indicating that the mean rotational variation of the sunspot numbers typically has one maximum and one minimum (overlaid by minor fluctuations). The wavelet analysis shows that spectral power for single periods varies for certain time intervals. A systematic drift during the solar activity cycle is not detected. Similar results are obtained from the superimposed epochs. Periodic "flip-flops" with time scales of a few years as for some stars are not found for the Sun in this investigation.

Conclusions. Sunspots are not distributed equally over the longitudes; there is a more active and a less active hemisphere. The rotation period derived from the pattern varies over long time scales. The results found in this work are not in favor of an explanation of the variations due to a differential rotation law. The rotation of the sunspot distribution pattern might reflect the internal rotation of the Sun, but it better fits the range of highest rotation rates in the upper convection zone than the rotation near the tachocline.
\end{abstract}

Key words. Sun: sunspots - Sun: rotation

\section{Introduction}

Solar activity as seen from Earth varies with the rotation of the Sun. Losh (1938) found that there is one maximum and one minimum per rotation, and that the phase of the maximum is stable over a solar cycle. Balthasar \& Schüssler (1984) investigated sunspot numbers and showed that the phase of the maximum remains more or less the same for two solar cycles and then it changes by about $180^{\circ}$. The result of Balthasar \& Schüssler is strongly influenced by the high activity in the maxima of the solar cycles. Such results are obtained from superimposed epoch analysis, where the choice of the rotation period is extremely important. The most persistent pattern is obtained using the (synodic) Bartels period of 27.00 days which was detected in the variation of geomagnetic indices (Chapman \& Bartels 1940). Assuming this period, Balthasar \& Schüssler (1983) show a good correlation between the sunspot distribution and the geomagnetic C9-index. Periods close to this one are detected in magnetograms (Bumba \& Howard 1969; Henney \& Harvey 2002), and in the interplanetary magnetic field (Svalgaard \& Wilcox 1975). Knaack \& Stenflo (2005) and Knaack et al. (2005) used magnetograms from Mt. Wilson and from Kitt Peak and found that the dominant rotation periods are different for cycles 21, 22 and 23. Bouwer (1992) investigated various indicators of solar activity and found that precise periods between 27 and 28 days persist only for a short time, sometimes only

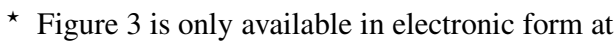
http: //www . aanda. org for a few solar rotations. Bai (2003) searched for locations with repetitive occurrence of flares. He called them "hot spots" and he found a persistence of decades, although the individual rotation rates of such hot spots are quite different. Using the Carrington period of 27.275 days which determines the heliographic longitudes, the pattern in the distribution of the sunspot numbers is destroyed after some time. Berdyugina \& Usoskin (2003) showed that the preferred longitude range drifts in phase, and this phase drift depends on the differential rotation. Taking the differential rotation into account, they find indications for two branches of active longitudes and a "flip-flop" between them with times scales of a few years. This result was doubted by Pelt et al. (2005). After refining their methods, Usoskin et al. (2005) and Berdyugina et al. (2006) confirmed the previous result. Usoskin et al. (2005) introduced a dynamic reference frame which rotates with the mean rate of the differential rotation with respect to the phase of the solar cycle. The latitudinal dependence of the determined rotation law corresponds to that of the deep convection zone $(0.75-0.8$ solar radii). Note that the authors published an erratum (Usoskin et al. 2007) that they did not consider the difference of the equatorial velocity and the angular velocity of the Carrington system in the first paper by Berdyugina \& Usoskin (2003). Nevertheless, Pelt et al. (2006) after a careful statistical analysis of the same data come again to the conclusion that a century-scale persisting longitudinal pattern does not exist.

Similar patterns are also detected in stars. At least ten stars exhibit a "flip-flop" of the preferred longitude range, first discovered by Jetsu et al. $(1991,1993)$ for the giant star FK Com and 

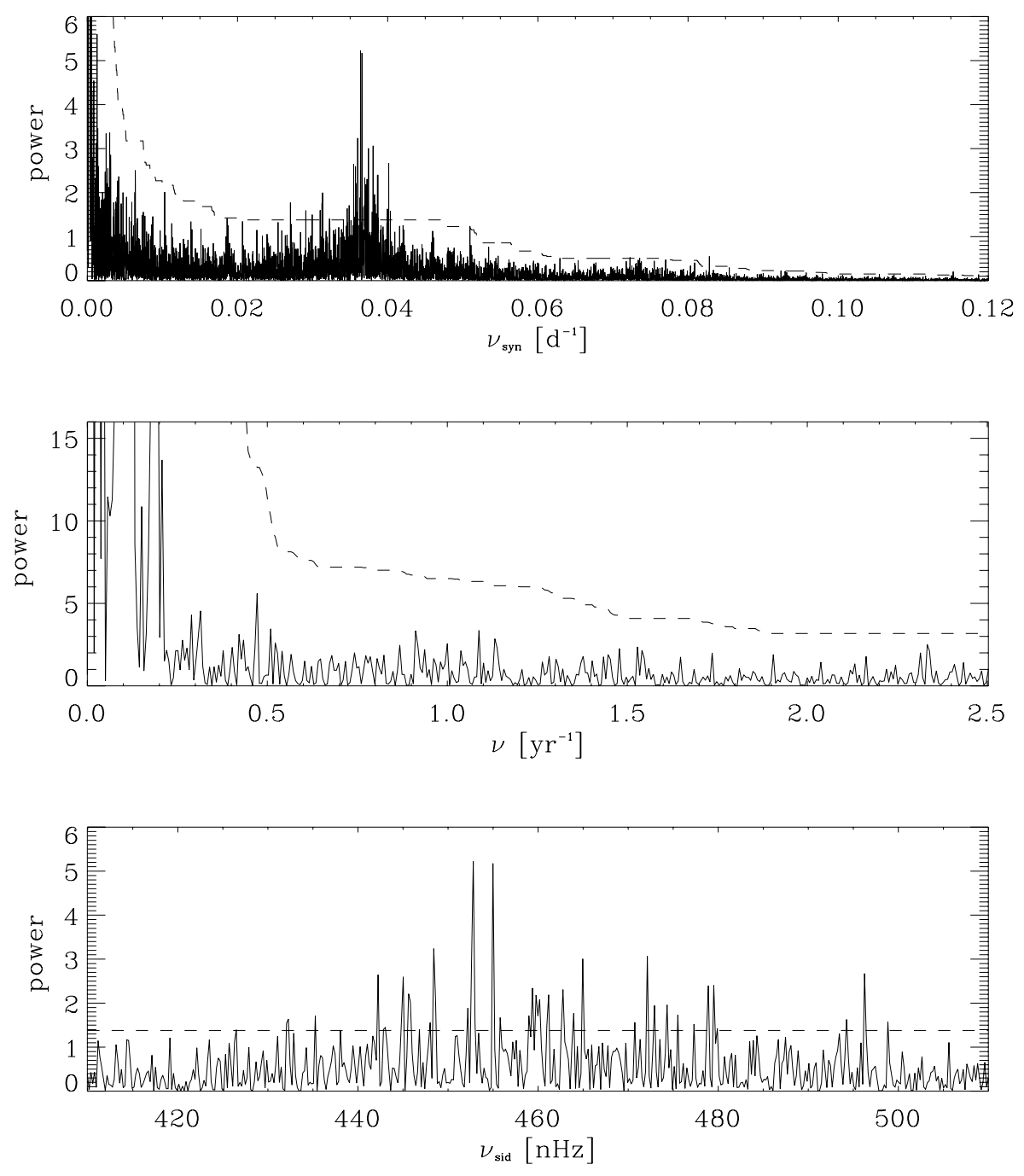

Fig. 1. Power spectrum of the daily sunspot numbers. The lower panels are extracts from the upper one, the middle one for periods around 1 year, the lower one shows the rotation range. The dashed line indicates the 99.99\%-significance level which is exceeded by one out of 10000 power values by chance. by Berdyugina \& Tuominen (1998) for RS CVn-stars. Korhonen et al. $(2002,2004)$ found that such "flip-flops" occur periodically, and for FK Com, the time between such events is about 3 years and the full cycle is about 6 years long. Berdyugina et al. (2002) found a "flip-flop" cycle of 5.2 years for the young solar analog LQ Hyd. Dynamo models including uneven modes to explain the preferred active longitudes are presented by Moss (2004, 2005), Bigazzi \& Ruzmaikin (2004), Elstner \& Korhonen (2005), Korhonen \& Elstner (2005) and Beryugina et al. (2006). Dikpati \& Gilman (2005) investigated MHD instabilities at the tachocline using a "shallow water"-model and find that such instabilities can be used to explain the occurrence of active longitudes. Fluri \& Berdyugina (2004) present ideas of how "flipflops" might be produced.

The aim of this work is to investigate the solar behavior. To compare with the stellar behavior, I prefer to use an integral measure of the solar activity. Such a measure with an extended time base is the radio flux at $10.7 \mathrm{~cm}$ which has been investigated by Mouradian et al. (2002) who found many peaks in the power spectrum between 25 and 29 days. Another measure that reveals the activity in the photospheric layers of the Sun is the daily sunspot number. The daily sunspot numbers for the time interval from 1818 through 2006 are provided by Van der Linden et al. (2006). Because of many gaps in the early part of this set, only data from 1848 onwards are used for most parts of the following analysis.

\section{The power spectrum}

Power spectra of the daily sunspot numbers have been presented by Knight et al. (1979) and Bogart (1982). Both papers report a broad peak for the solar rotation rate around 27 days and another peak close to 12 days. An extended study is presented by Henney \& Harvey (2002). They find a dominant peak at 27.03 days for the last two cycles, but somewhat different periods for earlier cycles. Here I present the power spectrum for an extended total period from January 1848 through December 2006. Including data before 1848 after filling gaps by a spline interpolation, the result changes somewhat, because gaps, even after filling them, introduce artifacts. Therefore it is better to avoid these early periods. The result is shown in Fig. 1.

Determing the significance levels, one has to consider that the appearance or disappearance of a sunspot group at the solar limb introduces steps into the data set leading to frequency dependent noise. Following Balthasar (2003), the slope of such a noise is estimated by a running mean over 101 power points (full box width) which is forced to decrease monotonically with frequency, i.e., if the running mean would increase at the next higher frequency point, it is set to the previous value. Regarding the power spectrum as the variance of the Fourier transform of the data, the ratio of the original power spectrum and the smoothed one should follow an f-distribution with 2 and 200 degrees of freedom. As significance criterion I choose a factor applied to the smoothed spectrum that one out of 10000 values 
Table 1. Synodic and siderial rotation periods and corresponding siderial frequencies. Sunspot rotation periods are calculated from $\omega=$ 14.551-2.87 $\sin ^{2}(B)$ determined by Balthasar et al. (1986). The value for the solar interior is the one just below the tachocline taken from Eff-Darwich et al. (2002).

\begin{tabular}{lccc}
\hline \hline Name & $P_{\text {syn }}[$ days $]$ & $P_{\text {sid }}[$ days $]$ & $v[\mathrm{nHz}]$ \\
\hline & 25.846 & 24.138 & 479.5 \\
spots at equator & 26.538 & 24.741 & 467.8 \\
spots at $15^{\circ}$ & 26.921 & 25.073 & 461.6 \\
Bartels & 27.000 & 25.141 & 460.4 \\
Carrington & 27.275 & 25.381 & 456.0 \\
1st high powerpeak & 27.361 & 25.454 & 454.7 \\
2nd high powerpeak & 27.491 & 25.567 & 452.7 \\
spots at 30 & 28.020 & 26.034 & 444.8 \\
& 28.200 & 26.179 & 442.1 \\
\hline interior & 28.698 & 26.607 & 435.0 \\
\hline
\end{tabular}

exceeds this level by chance (99.99\%-level). Note that there are 29037 values below the Nyquist frequency, thus one still would expect three cases of high peaks due to chance.

The dominant peak occurs at 10.6 years corresponding to the solar cycle. Smaller peaks close to half of cycle length are also present. These peaks are clipped in Fig. 1 since the aim of this paper is an investigation of solar activity patterns in relation to the solar rotation. Solar rotation shows up as a prominent hump of power peaks between synodic periods of 25.85 and 28.20 days corresponding to siderial frequencies between 440 and $480 \mathrm{nHz}$ (Periods derived directly from the relative numbers are synodic periods). Nevertheless none of these peaks fits exactly to the Carrington period of 27.275 days which is used to determine the heliographic longitudes. The two highest peaks in this range are at 27.361 and 27.491 days (454.7 and $452.7 \mathrm{nHz}$ ). Two weaker but still significant peaks are present near 28 days $(445 \mathrm{nHz})$, the rotation rate of spots at a latitude of about $30^{\circ}$, a period which is also detected for the solar corona (Sykora 1971). However, one should not put too much weight on the single peaks as modulation by the solar cycle splits the rotation signals. The dominant cycle length would lead to split rotation peaks shifted by $\pm 3 \mathrm{nHz}$ away from the original position.

The first harmonic of the rotation near $910 \mathrm{nHz}$ corresponding to $0.074 \mathrm{~d}^{-1}$ is only very weakly indicated, and it is clearly below the significance level. Peaks are one order of magnitude smaller than the rotation peaks. This means that the sunspot numbers vary with heliographic longitude and that there are only one dominant maximum and one dominant minimum which are $180^{\circ}$ apart. In case of a bi-modal distribution, one would expect higher power peaks near the overtone.

At 12.07 days $\left(v_{\text {syn }}=0.08285 \mathrm{~d}^{-1}\right)$ a single peak exceeds its neighbors, corresponding to the one reported by Knight et al. (1979) and Bogart (1982). This period is clearly shorter than half the rotation period. Peaks for longer periods than the rotation but shorter than the overtone of the solar cycle are rather small and clearly below the significance level. Especially, a period near 1.3 years does not show up.

\section{Wavelet analysis}

In order to search for temporal changes of the main rotation periods I performed a wavelet analysis for the daily sunspot numbers. Frick et al. (1997) presented a wavelet analysis for the monthly sunspot numbers, which is of course not sensitive to rotational changes. If the rotation rate of the pattern in the sunspot numbers is related to the differential rotation and the
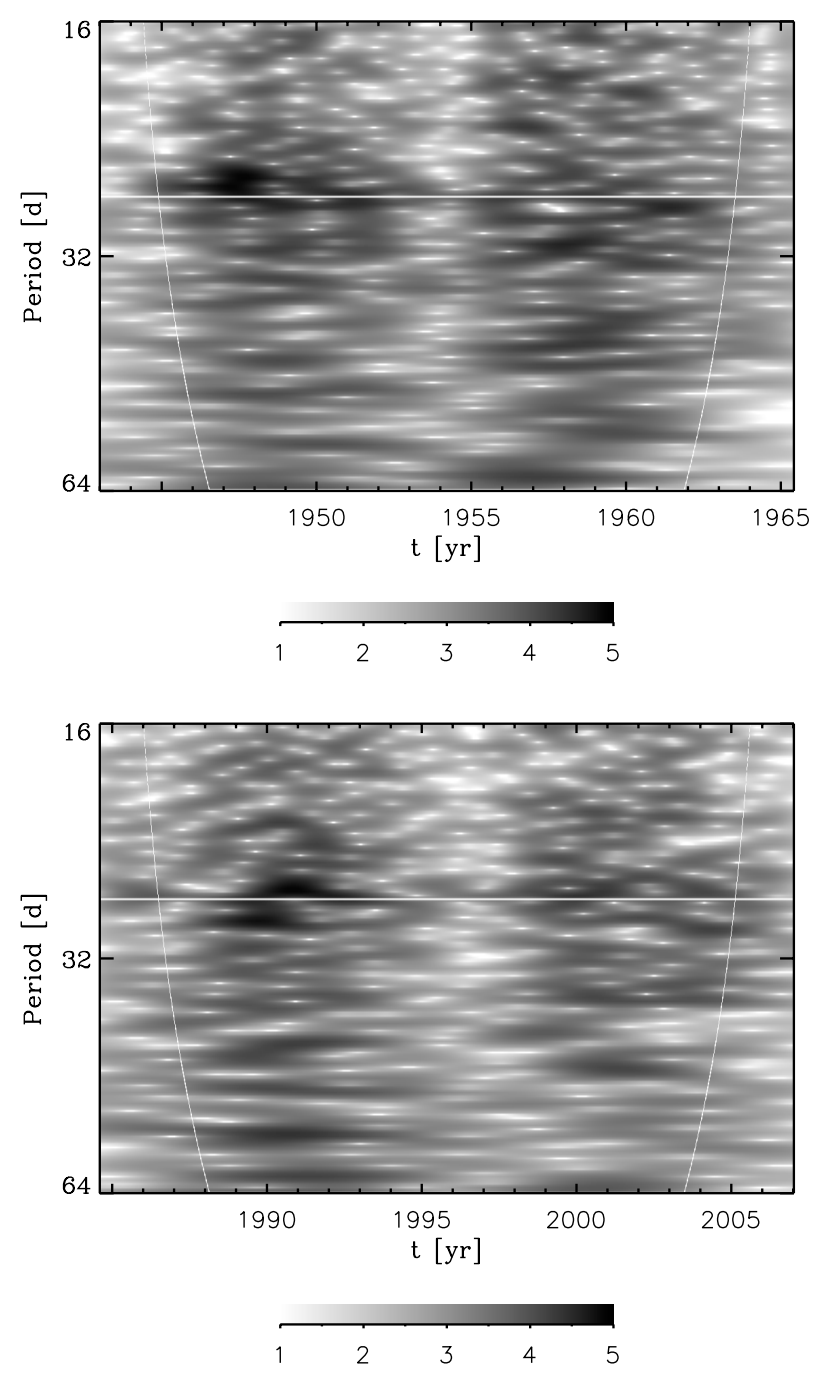

Fig. 2. Wavelet analysis for the cycle pairs 18 and 19 (1944-1965, upper panel) and 22 and 23 (1985-2006, lower panel). The decadic logarithm of the wavelet power is given; periods are synodic periods. The horizontal line marks the period of 27.0 days. The dotted line shows the cone of influence; values below are not reliable (see text).

equatorward drift of the activity belt (butterfly diagram), one would expect an increase of the angular velocity during the solar cycle.

The software was taken from Torrence \& Compo (1998). The wavelet type is Morlet, because it is commonly used, and, compared to the alternatives in the package, it contains more oscillations thus providing a better frequency resolution, as it is needed for the present investigation. I set the dimensionless parameter $\omega_{0}=72$, which is a measure for the number of oscillations within the wavelet itself. This value influences the frequency and time resolution of the analysis. I selected a clearly higher value than the choice of Knaack et al. (2005) in order to be able to distinguish between rotational periods roughly $0.4 \mathrm{~d}$ apart and to be still able to see in which phase of the solar activity cycle they occur. Applying the fast Fourier transform in the calculation, it is assumed that the data are cyclic. This introduces "wrap-around" effects at the edges of the data set, and the results there are not reliable. The boundary of the affected range is marked by the so-called "cone of influence" (see Torrence \& Compo 1998). Figure 2 shows as examples two pairs of solar activity cycle; , all wavelet power spectra for cycles 10-23 are 

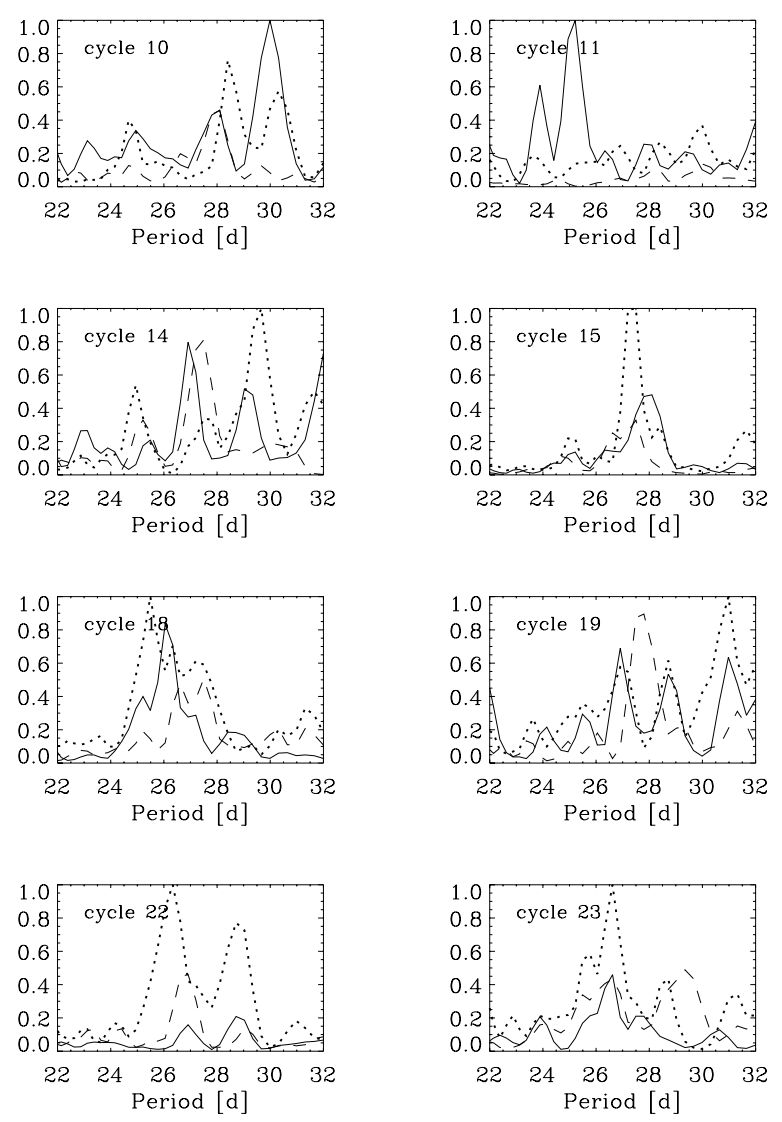
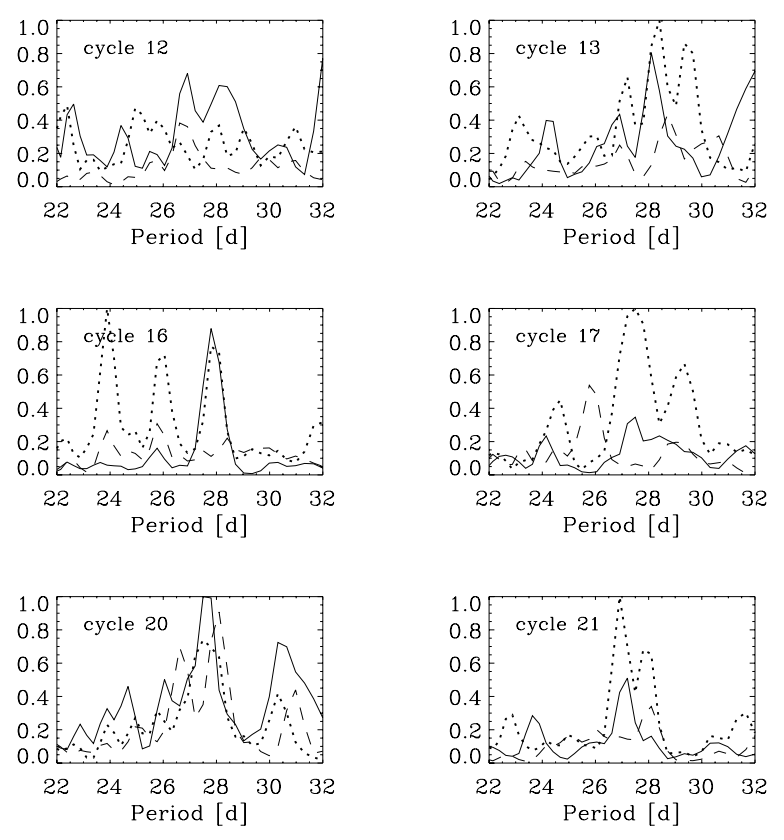

Fig. 4. Averages of the wavelet power spectra over 1000 days each in the rising phase (solid line), around maximum (dotted line) and in the decaying phase (dashed line) of the solar cycles.

electronically available as Fig. 3. Results for the other cycles are not significantly different, only somewhat noisier the older the data are and the lower the total activity of the cycle was.

As expected, there is enhanced power for the periods of rotation, and variations with time are visible. Assuming the rotation law of Usoskin et al. (2005), one would expect a decrease of the rotation period from about 29 days to 27 days during the solar cycle. Such an increase exists in cycle 15, see Figs. 3 and 4, but it is not detectable for the other included solar cycles, or it is overlaid by other stronger signals. This result is not in agreement with the expectation for the case that the rotation of the pattern of the sunspot numbers follows the differential rotation with the drift of the activity belt towards the equator (butterfly diagram).

\section{Superimposed epoch analysis}

Phase shifts become best visible in a superimposed epoch graph. Yearly averages are calculated keeping the phase with respect to a preselected period. Figures 5 and 6 show such graphs for the Bartels period and the period of one of the two highest power peaks (27.491 days) in the rotation range. A sine-like variation occurs during solar rotation for both periods shown as well as for many others that have been investigated. Therefore, sine-fits are determined for all yearly curves. Their variable part is almost free from the longterm modification of the solar cycle, and they are also given in the figures. If the selected period is correct, the difference between minimum and maximum should be largest, and the shape of the curves should not move in phase. The shape of the curves drifts to the right if the selected period is slightly too short, and to the left if it is slightly too long.
In Figs. 5 and 6, drifts of the pattern to the left and to the right are present. This indicates that the dominant period varies with time. "Flips" and "flops" happen, but not on a periodic time scale. The most stable phase positions for the maxima are found for the period of 29.491 days, one of the two highest power peaks, i.e. the maximum appears often at phase zero and the minimum near phase 0.5 . There are short periods with a different maximum phase, a flip of phase is present after 1980. Between 1925 and 1930, a drift of the phase to the right is present which indicates an even slower rotation. A drift to the left is seen between 1955 and 1960.

Looking at the temporal variations of the maxima of the fits, the highest average value is found for the Bartels period, although single higher values occur for other periods. However, the differences between the various periods are too small to be used as a clear indicator of a preferred period.

Figure 7 shows averages of the rotational variations within the Bartels period and averages of the sine fits. In many cases the curves are quite parallel for two subsequent cycles, beginning with an even numbered one. This kind of pair-building of two cycles is the same as the one suggested by Gnevyshev \& Ohl (1948), who claimed that the activity of the subsequent odd cycle is correlated with the previous even cycle, and the odd cycle shows higher activity. For this averages, the first two and the last three years of the cycle are omitted. For cycles 12 through 19 , the maximum occurs at nearly the same phase for an even and the subsequent odd cycle, then it shifts to the opposite phase until the next even cycle, as was found by Balthasar \& Schüssler (1984). However the latest added cycles do not fit this behavior. Correlation coefficients between the curves of two subsequent cycles alternate between significant positive and negative values 


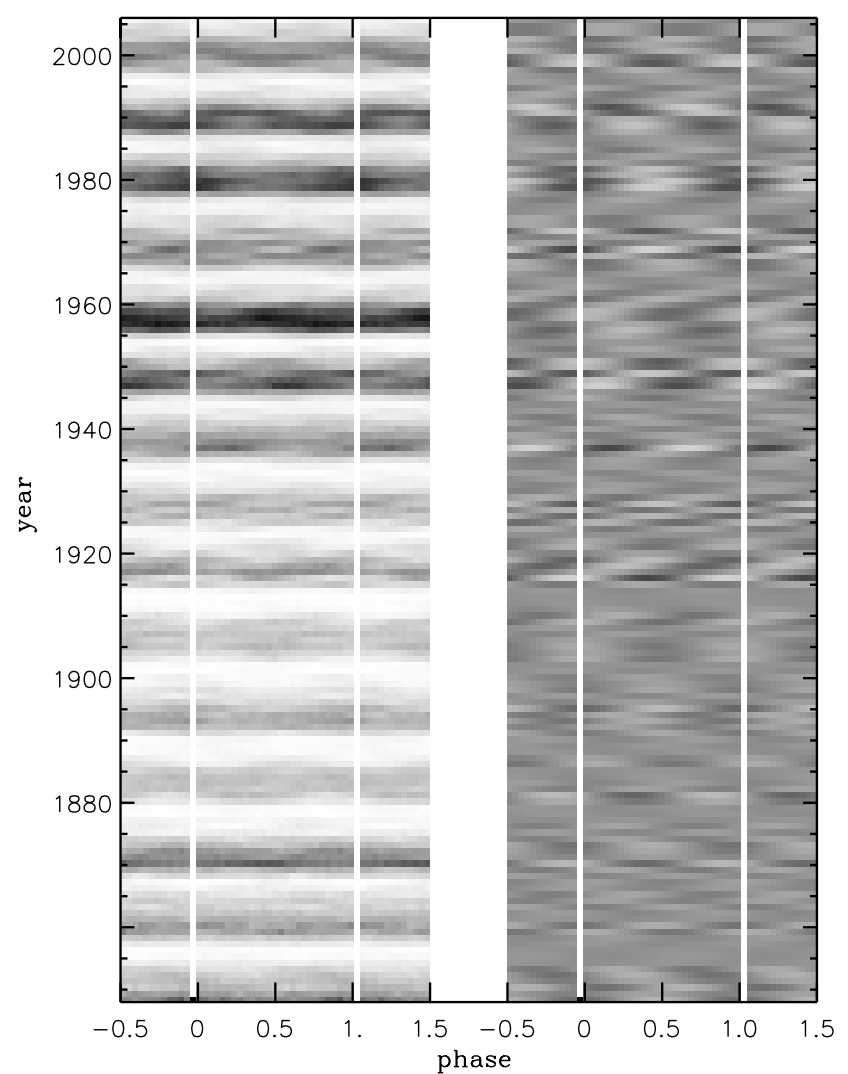

Fig. 5. Phase dependence of the sunspot numbers for the Bartels period (27.00 day synodic), averaged for single years. The left column represents the data themselves and the right column the varying part of a sine-fit.

for these seven cycles. Taking two more years of the cycle into account, the relation between the cycles changes in the sense that there is less systematic correlation. Using another preselected period, one obtains in some cases even higher correlation coefficients between two cycles, but no systematic variation of them.

\section{Discussion}

\subsection{Relation to differential rotation}

From sunspot positions it is measured that they rotate differentially. Balthasar et al. (1986) determined $\omega(\psi)=$ $14.551-2.87 \sin ^{2}(\psi)$ [degrees/day], where $\omega$ is the sideric angular velocity and $\psi$ the heliographic latitude. The error of the factor of the second term is only 0.06 , so this value is not in agreement with the value $-3.46 \pm 0.06$ used by Berdyugina \& Usoskin (2003). Using this equation, the Bartels period corresponds to a latitude of $16 .^{\circ} 52$ and the Carrington period to a latitude of $20{ }^{\circ} 95$. Roughly for a latitude difference of $20^{\circ}$ (crudely the width of latitude belt of activity) we deal with a difference of about $0.5^{\circ} \mathrm{d}^{-1}$ for the rotation velocities. Thus differential rotation builds up a phase difference of $180^{\circ}$ between upper and lower edge of the activity belt over one year. However, the distribution of sunspots is not equal over the whole width of the belt, so an active longitude is not smeared out completely within one year. Since the latitude belt of the sunspot activity migrates towards the equator during the solar cycle, one would expect a rotational speedup for the distribution pattern of sunspots. Such a speedup would cause a phase drift in Figs. 5 and 6 in the sense that the maximum of the distribution occurs at earlier phases.

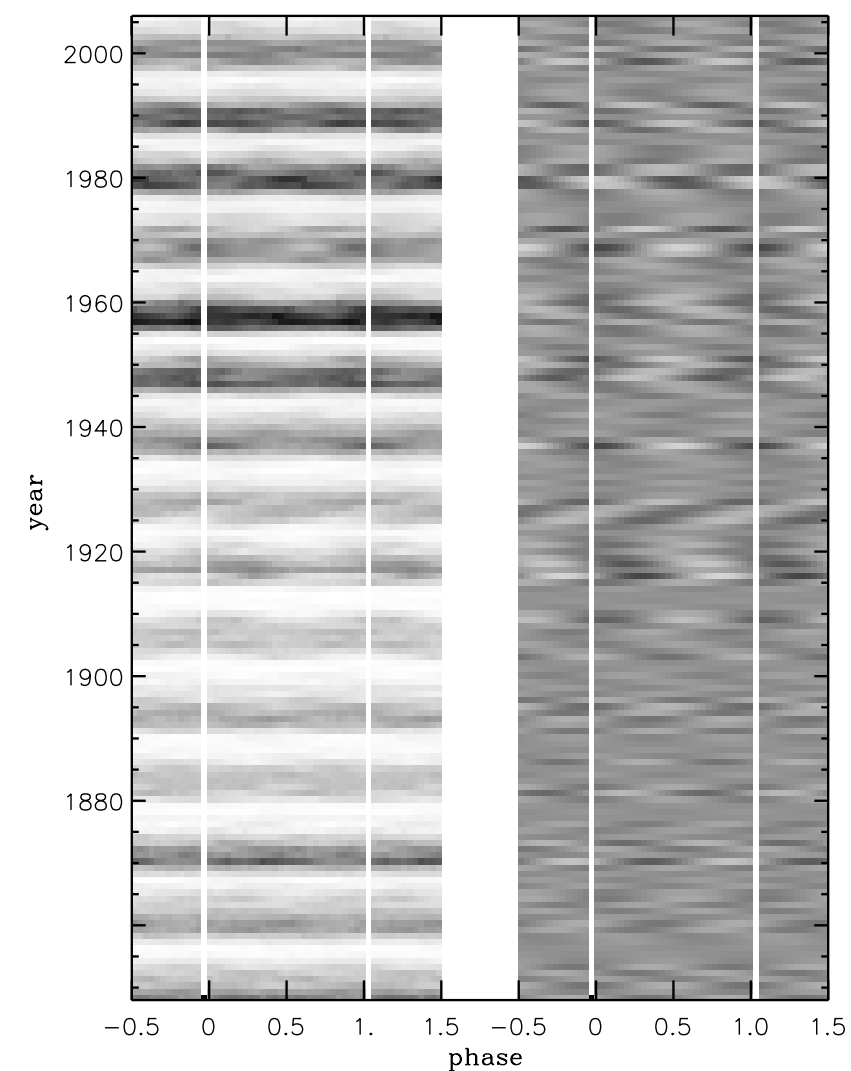

Fig. 6. Same as Fig. 5, but now for the period of the second high rotation power peak (27.491 d).

This is perhaps present for a few cycles, but not in a systematic way for the majority of all cycles. Thus the distribution pattern is not well correlated with the differential rotation.

\subsection{Relation to internal rotation of the Sun}

The distribution pattern could represent the solar rotation in deeper layers. Helioseismology allows us to measure the internal solar rotation, and results were recently published by EffDarwich et al. (2002) and Schou et al. (2002). As Beck (1999) pointed out, the observed rotation rate of sunspots corresponds to the internal rate near 0.93 solar radii. The solar dynamo is generally expected to work close to the tachocline layer, where the differential rotation of the convective zone transits into a rigid rotation. The sidereal rotation frequency is about $430 \mathrm{nHz}$ there, a value that is about $30 \mathrm{nHz}$ slower than the rotation rates for the distribution pattern. Brandenburg (2005) presents this argument too, and also some others in favor of a dynamo working in the shear layer near the solar surface, although strong turbulent distortions and the short time for the shear to act argue against it.

The results of this paper as well as others lead to the idea that sunspots are not fixed in a very distinct layer inside the Sun; they might represent higher or deeper layers of the convection zone. If so, it is not surprising that it is almost impossible to determine a precise and persistant rotation rate from the distribution of sunspots. I cannot rule out that the smearing out of the pattern with time is caused by differential rotation, although the result of the wavelet analysis is not in favor of such an explanation.

No prominent power peak occurs near 1.3 years, the period found for variations of the rotation speed at the tachocline, see Howe et al. (2000) and Krivova \& Solanki (2002). 


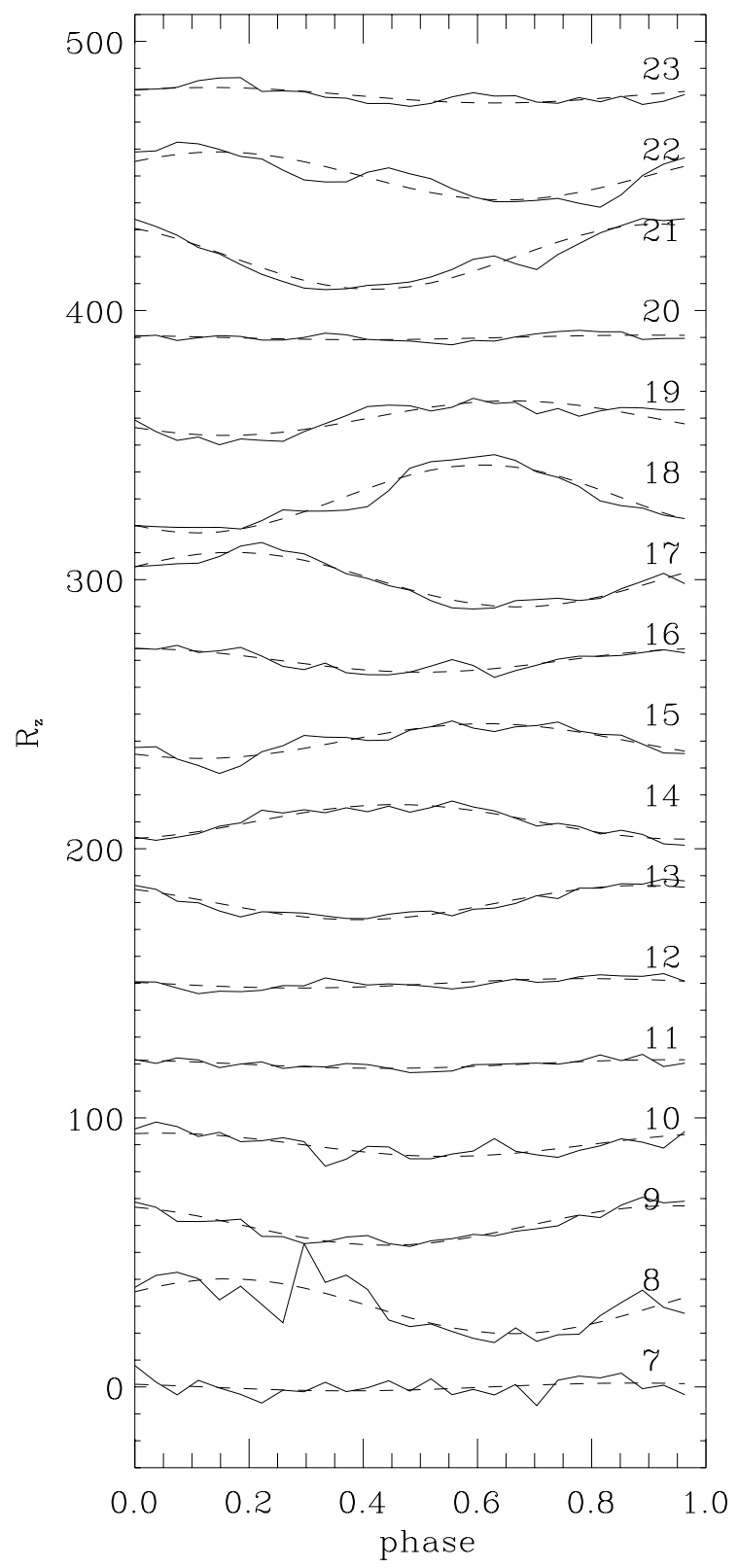

Fig. 7. Variations within the Bartels period, averaged over the maximum period of one solar cycle. The solid lines are the measured distributions, and dashed lines represent the sine fits. The number on the right is the number of the solar cycle. For better visibility, curves are shifted by multiples of 30 .

\subsection{Relation to stellar observations}

Stellar "flip-flops" were first found for FK Com by Jetsu et al. (1991, 1993), and the periodicity of this phenomenon was discovered by Korhonen et al. $(2001,2002)$ and Berdyugina et al. (2002). The Sun has a more and a less active hemisphere. Sometimes a rapid exchange of the two hemispheres happens, but an over a long time persisting periodic "flip-flop" is not detected for the Sun for periods near 3.6 years or for any other period of a few years. A period of four activity cycles is indicated for the distribution around the activity maxima of seven cycles assuming the Bartels period, but data before and after do not fit it well (see Fig. 7). Therefore the conclusion of Balthasar \& Schüssler (1984) cannot be kept as a generally valid one.

\section{Conclusions}

From the results above I draw the following conclusions.

1. The pattern of solar activity as seen from Earth exhibits a variation with solar rotation. There is a dominant bi-modal distribution with one maximum and one minimum that are $180^{\circ}$ apart. This behavior is comparable to that recently discovered for certain stars. If there was a second maximum, the overtone in the power spectrum should appear.

2. There is no single period in the pattern that is constant over the whole time interval 1848-2006. Using the Bartels period and restricting to the years around the maxima of the solar cycles, the phase position of the pattern remains for two solar cycles, then it changes by about $180^{\circ}$. This sequence holds at least for cycles 12-19.

3. A periodic "flip-flop" with time scales of about 3.6 years or a similar period is not detected in this investigation.

4. If the distribution of the sunspot numbers depends on the observed differential rotation obtained from individual sunspot groups or from helioseismology, one would expect a systematic drift in period over the solar cycle in the wavelet analysis or in phase in the superimposed epoch analysis. Such a drift is not observed.

5. The pattern of sunspot numbers could represent the rotation in layers where the spots are formed. It is generally assumed that the solar dynamo works in layers close to the tachocline, but the rotation frequency there is too low by about $30 \mathrm{nHz}$. The observed rotation rates fit better to those of the convection zone between 0.9 and 0.95 solar radii.

These results do not rule out a hidden, more systematic interaction of rotation, convection and the dynamo process in the Sun. Improved models that indicate where sunspots are formed and anchored are needed for a better understanding of the longitudinal distribution of the solar activity.

Acknowledgements. I would like to thank my colleagues R. Arlt, H.E. Fröhlich, H. Korhonen and M. Küker for fruitful discussions. The comments of the anonymous referee helped to improve the manuscript. The daily sunspot numbers were provided by Solar Influence Data Analysis Center (SIDC)at URL: http://sidc. oma. be. Wavelet software was provided by C. Torrence and G. Compo, and is available at URL: http://atoc.colorado.edu/research/ wavelets/

\section{References}

Bai, T. 2003, ApJ, 585, 1114

Balthasar, H. 2003, Sol. Phys., 218, 85

Balthasar, H., \& Schüssler, M. 1983, Sol. Phys., 87, 23

Balthasar, H., \& Schüssler, M. 1984, Sol. Phys., 93, 177

Balthasar, H., Vázquez, M., \& Wöhl, H. 1986, A\&A, 155, 87

Beck, J. G. 1999, Sol. Phys., 191, 47

Berdyugina, S. V., \& Tuominen, I. 1998, A\&A, 336, L25

Berdyugina, S. V., \& Usoskin, I. 2003, A\&A, 405, 1121

Berdyugina, S. V., Pelt, J., \& Tuominen, I. 2002, A\&A, 394, 505

Berdyugina, S. V., Moss, D., Sokoloff, D., \& Usoskin, I. G. 2006, A\&A, 445, 703

Bigazzi, A., \& Ruzmaikin, A. 2004, ApJ, 604, 944

Bogart, R. 1982, Sol. Phys., 76, 155

Bouwer, S. D. 1992, Sol. Phys., 142, 365

Brandenburg, A. 2005, ApJ, 625, 539

Bumba, V., \& Howard, R. 1969, Sol. Phys., 7, 28

Chapman, S., \& Bartels, J. 1940, Geomagnetism (Oxford: Clarendon)

Dikpati, M., \& Gilman, P. A. 2005, ApJ, 635, L193 
Eff-Darwich, A., Korzennik, S. G., \& Jiménez-Reyes, S. J. 2002, ApJ, 573, 857 Elstner, D., \& Korhonen, H. 2005, AN, 326, 278

Fluri, D., \& Berdyugina, S. V. 2004, Sol. Phys., 224, 153

Frick, P., Galyagin, D., Hoyt, D. V., et al. 1997, A\&A, 328, 670

Gnevyshev, M. N., \& Ohl, A. I. 1948, AZh, 25, 19

Henney, C. J., \& Harvey, J. W. 2002, Sol. Phys., 207, 199

Howe, R., Christensen-Dalsgaard, J., Hill, F., et al. 2000, Science, 287, 2456

Jetsu, L., Pelt, J., Tuominen, I., \& Nations, H. L. 1991, in The Sun and Cool Stars: Activity, magnetism and dynamos, ed. I. Tuominen, D. Moss, \& G. Rüdiger (Heidelberg: Springer-Verlag), Proc. IAU Coll., 130, 381 Jetsu, L., Pelt, J., \& Tuominen, I. 1993, A\&A, 278, 449

Knaack, R., \& Stenflo, J. O. 2005, A\&A, 438, 349

Knaack, R., Stenflo, J. O., \& Berdyugina, S. V. 2005, A\&A, 438, 1067

Knight, J. W., Schatten, K. H., \& Sturrock, P. A. 1979, ApJ, 227, L153

Korhonen, H., \& Elstner, D. 2005, A\&A, 440, 1161

Korhonen, H., Berdyugina, S. V., Strassmeier, K. G., \& Tuominen, I. 2001, A\&A, 379, L30

Korhonen, H., Berdyugina, S. V., \& Tuominen, I. 2002, A\&A, 390, 179
Korhonen, H., Berdyugina, S. V., \& Tuominen, I. 2004, AN, 325, 402 Krivova, N. I., \& Solanki, S. K. 2002, A\&A, 394, 701

Losh, H. M. 1938, Publ. Michigan, 7, 127

Moss, D. 2004, MNRAS, 352, L17

Moss, D. 2005, A\&A, 432, 249

Mouradian, Z., Bocchia, R., \& Botton, C. 2002, A\&A, 394, 1103

Pelt, J., Tuominen, I., \& Brooke, J. M. 2005, A\&A, 429, 1093

Pelt, J., Brooke, J. M., Korpi, M. J., \& Tuominen, I. 2006, A\&A, 460, 875

Schou, J., Howe, R., Basu, S., et al. 2002, ApJ, 567, 1234

Svalgaard, L., \& Wilcox, J. M. 1975, Sol. Phys., 41, 461

Sykora, J. 1971, Sol. Phys., 18, 72

Torrence, C., \& Compo, G. P. 1998, Bull. Am. Met. Soc., 79/1, 61; see also: http://atoc. colorado. edu/research/wavelets/

Usoskin, I. G., Berdyugina, S. V., \& Poutanen, J. 2005, A\&A, 441, 347

Usoskin, I. G., Berdyugina, S. V., \& Poutanen, J. 2007, A\&A, 464, 761 (Erratum)

Van der Linden, R. A. M., \& the SIDC team, Online catalogue of the sunspot index, http://sidc.oma.be/html/sunspot.html 
H. Balthasar: Rotational periodicities in sunspot relative numbers, Online Material $p 1$

\section{Online Material}


H. Balthasar: Rotational periodicities in sunspot relative numbers, Online Material $p 2$
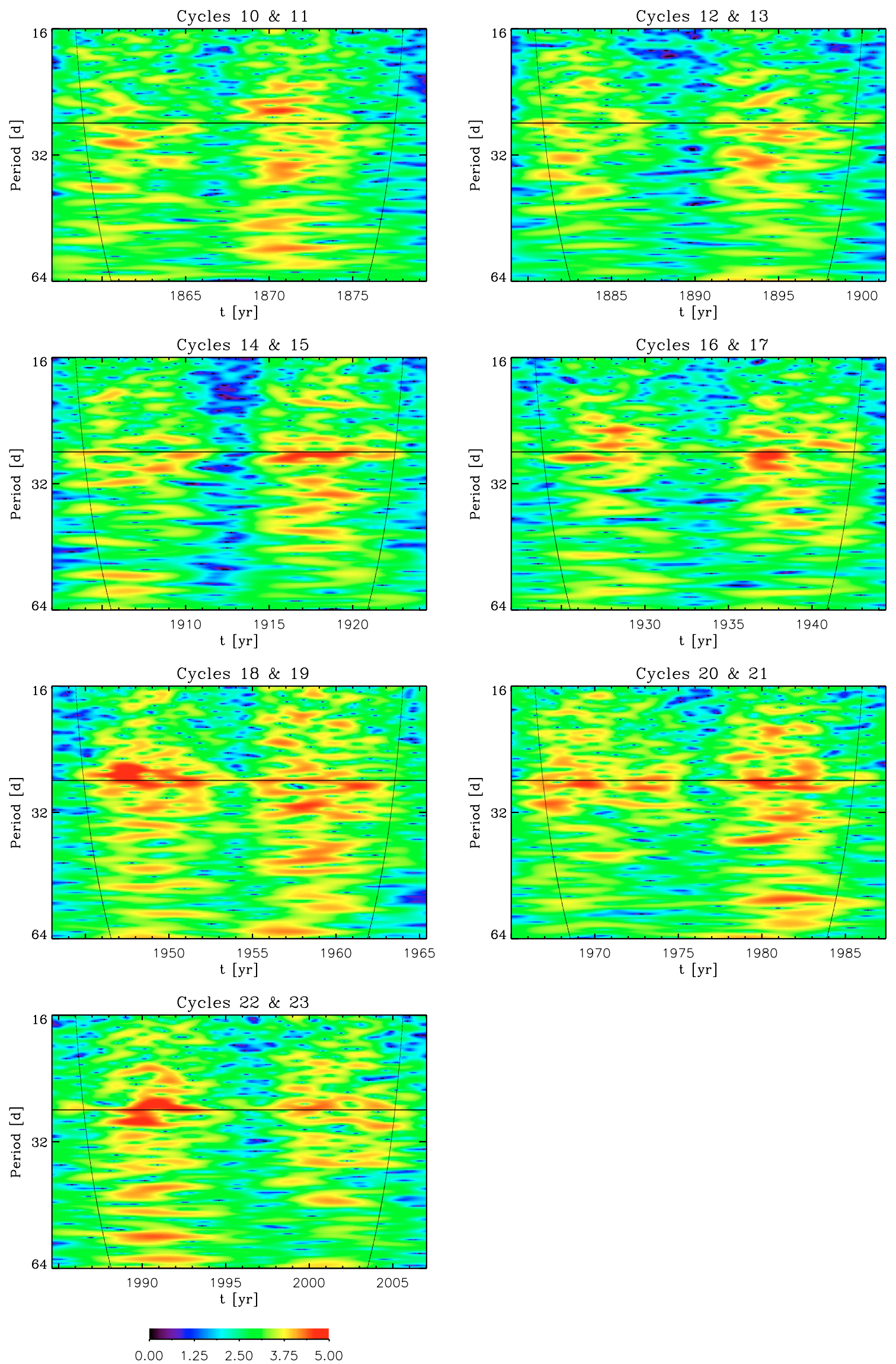

Fig. 3. Wavelet analysis as in Fig. 2 for cycle pairs from cycle 10 through 23 in color coding. The decadic logarithm of the wavelet power is given; periods are synodic periods. The horizontal line marks the period of 27.0 days. The dotted line shows the cone of influence; values below are not reliable (see text). 